\title{
Causes of vision impairment and assessment of need for low vision services for students of blind schools in Nepal
}

\author{
Kansakar I ${ }^{1}$, Thapa HB $^{1}$, Salma KC ${ }^{1}$, Ganguly $\mathbf{S}^{1}$, Kandel RP' ${ }^{2}$, Rajasekaran $\mathbf{S}^{1}$ \\ ${ }^{1}$ Lumbini Eye Institute, ${ }^{2}$ Seva Foundation, Seva Canada Society, Nepal
}

\begin{abstract}
Background: The present study is first of its kind to evaluate causes of visual impairment of blind students in Nepal and assess their need for low vision rehabilitation services.

Aim: To evaluate causes of vision impairment of students enrolled in blind schools in Nepal and assess the need for low vision rehabilitation services in these students.

Materials and methods: A survey was conducted in 12 blind schools in Nepal, which were registered with Nepal Association for Welfare of Blindness (NAWB).It was conducted by a team of an ophthalmologist and an optometrist, by using standard eye examination protocols of the World Health Organization Prevention of Blindness Program (WHO/ PBL).

Results: Of the 345 students enrolled in 12 schools, 285 students were examined (response rate of $82.61 \%$ ). The students were in the 5 - 29 years age group. Nearly three-fourth of the children had become blind within one year of age and $52.3 \%$ visually impaired at birth and $20.7 \%$ developed vision impairment within one year of age. After refraction, 26 students $(9.12 \%)$ had mild visual impairment, 21 students $(7.37 \%)$ had severe visual impairment and 238 students $(83.51 \%)$ were blind. The main cause of vision impairment was found to be corneal $35.79 \%$ and retina diseases, mainly dystrophy, $20.35 \%$ followed by problems with the whole globe, lens and optic nerve, accounting for $13.33 \%, 12.63 \%$ and $12.98 \%$ respectively. The major etiological factors were those of childhood such as Vitamin A deficiency, measles and similar causes $(42.11 \%)$ followed by hereditary causes $(25.26 \%)$. Of the total students examined, $48.07 \%$ were visually impaired due to preventable causes and $16.14 \%$ treatable aggregating to $64.21 \%$ of avoidable blindness. Fifty seven (28.22\%) students could read smaller than $2 \mathrm{M}$ print size after low vision assessment for near and 33(15.78\%) students benefited with telescopic trial for distance low vision.

Conclusion: In Nepal, renewed focus on providing best possible quality of life for visually impaired children by proper low vision assessment and eye health education focusing on, general public and community health workers, with governmental and institutional support is required to achieve Vision 2020 objectives to decrease childhood blindness.
\end{abstract}

Key words: childhood blindness, Nepal, blind school study, low vision, vision impairment

Tn the world today, a child goes blind every minute. 1 These children have a lifetime of blindness ahead, with an estimated 75 million blind-years (number blind $\mathrm{x}$ length of life), second only to cataract ${ }^{1}$. Blindness in childhood has negative impact on development of cognitive and social skills. It is estimated that out of the 1.4 Million blind children world-wide, one Million live in Asia ${ }^{1}$. Childhood blindness prevalence was estimated at 0.08 for the South East Asian Region (SEARO) that comprised of Nepal, India, Pakistan and Bangladesh ${ }^{2}$. As of 2006, there were an estimated 9.70 Million children aged below 15 years in Nepal accounting for $37.46 \%$ of the total population ${ }^{3}$. Nation-wide population-based studies on childhood blindness are relatively difficult as the target population is spread over a large geographical area. Therefore, the present study was conducted amongst students enrolled in blind schools in Nepal.

\section{Materials and methods}

Twelve out of 16 schools for enrolling blind children were selected for survey based on accessibility to transport and communication. Three clinical teams, each consisting of an ophthalmologist and an optometrist, conducted clinical examination and instituted detailed questionnaires, in the premises of the respective schools following strictly WHO/PBL eye examination protocol; Eye Examination Record for Children with Blindness and Low Vision (ERCB) 4 .

\footnotetext{
Correspondence

Dr. Irina Kanskar

Consultant Ophthalmologist,

Lumbini Eye Institute

Bhairahawa, Siddharthanagar -3

Lumbini, Nepal

E-mail: irakera@yahoo.com
} 
In each clinical team, the ophthalmologist took the history and performed eye examination to locate the site of abnormality leading to visual loss (anatomical classification), etiological classification of visual loss, diagnosis and formulation of future course of action required in addition to vision prognosis. The ophthalmologist also performed anterior segment examination using magnifying loupe and torch, while posterior segment examination was carried out using standard Heine direct ophthalmoscope with dilated fundus wherever indicated.

The optometrist recorded personal details of the child, visual assessment, general assessment, refraction \& evaluated need for low vision aids. Distance visual acuity was recorded with Bailey Lovie Chart (log MAR chart) in external illumination at a distance of three meters. Distance visual acuity was measured separately for each eye and both eyes together. Lighthouse near visual acuity chart was used to test near visual acuity.

Refraction was done only in cases where, in the optometrist's judgment, visual improvement could be expected. Best corrected distance and near acuity of each eye after refraction using Heine streak retinoscope were recorded. LV Prasad Eye Institute (LVPEI)'s low vision kit for eye care professionals in developing countries was used for low vision assessment.

Data was collected on standard World Health Organization data forms and analyzed using SPSS, standard statistical software. Patient identity was protected by adopting appropriate security procedures in the collection, storage and analysis.

\section{Results}

A total number of 285 students out of 345 enrolled in 12 blind schools were examined. The mean age of the students was 14.41 years having a standard deviation of 3.69. The visual status of blind school students at presentation and after refraction is shown in the table below (Table 1). After refraction seven students visual status improved from category of blindness.

Regarding onset of visual impairment, 52.3\% were visually impaired at birth and another $20.7 \%$ developed visual impairment within one year of age. Thus close to three-fourths $(73.00 \%)$ of the total children had become blind within one year of age.

\section{Anatomical Site of Abnormality Causing .Vision Loss:}

Pie diagram depicts anatomical locations for causes of visual loss (Fig 1).
The main diseases in the top five anatomical sites of visual loss were as follows:

1) In the 102 children with abnormality in cornea / phthisis, 33 (11.58\%) had abnormality in phthisis, $28(9.82 \%)$ scar, 27 (9.47\%) staphyloma, seven $(2.46 \%)$ keratoconus, six $(2.11 \%)$ dystrophy and one $(0.35 \%)$ other opacity.

2) In the 58 children with abnormality in retina, 47 (16.49\%) had dystrophy, one $(0.35 \%)$ each albinism and retinopathy of prematurity and nine $(3.16 \%)$ other causes.

3 ) In the 38 children with whole globe abnormality, $22(7.72 \%)$ had microphthalmos, 10 (3.51\%) buphthalmos, three $(1.05 \%)$ each of anophthalmos and glaucoma.

4) In the 37 children with abnormality in the optic nerve, $34(11.93 \%)$ had atrophy and three $(1.05 \%)$ had hypoplasia.

5) In the 36 children with abnormality in the lens, 22 $(7.72 \%)$ had cataract, ten $(3.51 \%)$ had aphakia and four $(1.40 \%)$ other causes.

\section{Etiological Categories of visual impairment}

Regarding the etiology of visual impairment diagram below is self explanatory (Fig 2).

Amongst 120 children with postnatal / infancy / childhood factors, $30(10.53 \%)$ had Vitamin A deficiency, 29 (10.18\%) meningitis, 21 (7.37\%) harmful traditional eye medicine (HTEM), 14 (4.91\%) measles, ten $(3.51 \%)$ trauma, six $(2.11 \%)$ encephalitis, five $(1.75 \%)$ pyrexia of unknown origin, two $(0.70 \%)$ each of amblyopia and typhoid and one $(0.35 \%)$ neoplasm.

Amongst the 72 children with hereditary causes, $23(8.07 \%)$ were auto recessive, nine (3.16\%) had autosomal dominance and could not be specified for 40 (14.04\%) children.

Of the 16 children blind due to intrauterine factors, $11(3.86 \%)$ had rubella, two $(0.70 \%)$ each due to toxoplasmosis and drugs / alcohol and one (0.35\%) disc anomaly. Of the nine children blind due to perinatal / neonatal factors, seven $(2.46 \%)$ had ophthalmia neonatorum and two $(0.70 \%)$ had retinopathy of prematurity.

Etiology could not be determined in 68 children of whom $29(10.18 \%)$ had cataract, 21 (7.37\%) abnormality since birth, $13(4.56 \%)$ glaucoma / buphthalmos, three $(1.05 \%)$ retinoblastoma and two $(0.70 \%)$ uveitis.

Avoidable Causes of Blindness: Of the total 285 children examined, 183 (64.21\%) children were blind 
due to avoidable causes, out of which $137(48.07 \%)$ were preventable and $46(16.14 \%)$ treatable conditions.

Additional Findings: Five (1.75\%) were found to be children of consanguineous marriage. Sixty nine (24.21\%) children showed positive family history for conditions such as congenital cataract, congenital glaucoma, retinal dystrophy, microphthalmos and albinism. The most frequent additional disabilities were hearing loss $(2.1 \%)$ followed by mental retardation $(1.1 \%)$ and physical handicap $(0.4 \%)$. Majority of surgical interventions already performed were for cataract $(11.56 \%)$, glaucoma $(2.10 \%)$ and increasing size of visual axis by optical iridectomy $(0.7 \%)$.

Low Vision Status: Out of 209 children with functional low, after distance correction nine $(4.03 \%)$ students could read $1 \mathrm{M}$ print size without low vision device.
Telescopic trial was done for all the 33 students who required distance correction and results are shown in the table below (Table 2).

The results of low vision assessment for near are shown in table below (Table 3). In total, 57( 48+9) $(27.27 \%)$ students could read smaller than $2 \mathrm{M}$ print size after low vision assessment including previous nine students who could read $1 \mathrm{M}$ print size after distance correction which means these students can read normal school text books (Table 3).

Therapeutic Interventions Advised: Of the total 285 students examined, spectacles for refractive error correction was recommended for $48(16.84 \%)$ students, low vision aids for another $62(21.75 \%)$ students and surgical intervention (cataract surgery and optical iridectomy in selected cases) for ten (3.51\%) students.

Table 1: Vision status at presentation and after refraction

\begin{tabular}{|c|c|c|c|c|c|c|c|c|c|}
\hline \multirow{3}{*}{$\begin{array}{l}\text { Best Corrected } \\
\text { Visual Acuity }\end{array}$} & \multirow{3}{*}{$\begin{array}{c}\text { WHO } \\
\text { Categories }\end{array}$} & \multicolumn{4}{|c|}{ At Presentation } & \multicolumn{4}{|c|}{ After Refraction } \\
\hline & & \multicolumn{4}{|c|}{ Age Groups } & \multicolumn{4}{|c|}{ Age Groups } \\
\hline & & $<=15$ & $>15$ & Total & $\%$ & $<=15$ & $>15$ & Total & $\%$ \\
\hline $6 / 6$ to $6 / 18$ & $\mathrm{NI}$ & 0 & 0 & $\mathbf{0}$ & 0.00 & 0 & 0 & $\mathbf{0}$ & 0.00 \\
\hline$<6 / 18$ to $6 / 60$ & VI & 15 & 5 & 20 & 7.02 & 21 & 5 & 26 & 9.12 \\
\hline$<6 / 60$ to $3 / 60$ & SVI & 16 & 4 & 20 & 7.02 & 15 & 6 & 21 & 7.37 \\
\hline$<3 / 60$ to $\mathrm{PL}$ & $\mathrm{BL}$ & 99 & 70 & 169 & 59.30 & 94 & 68 & 162 & 56.84 \\
\hline NPL & $\mathrm{BL}$ & 40 & 36 & 76 & 26.67 & 40 & 36 & 76 & 26.67 \\
\hline \multirow[t]{2}{*}{ Total } & No. & 170 & 115 & 285 & 100.00 & 170 & 115 & 285 & 100.00 \\
\hline & $\%$ & 59.65 & 40.35 & 100.00 & & 59.65 & 40.35 & 100.00 & \\
\hline
\end{tabular}

Table 2: Low Vision Assessment - Either Eye - Distance *

\begin{tabular}{|c|c|c|}
\hline & Frequency & Percentage $^{\wedge}$ \\
\hline $6 / 5-6 / 18$ & 19 & $9.09 \%$ \\
\hline Less than 6/18-6/60 & 14 & $6.69 \%$ \\
\hline Total & $\mathbf{3 3}$ & $\mathbf{1 5 . 7 8 \%}$ \\
\hline
\end{tabular}

* Assessed with LVA, VA with LVA

$\wedge$ As a percentage of the total 209 students with functional low vision

Table 3: LVA Assessment - Either Eye - Near *

\begin{tabular}{|c|c|c|}
\hline & Frequency & Percentage $^{\wedge}$ \\
\hline$<2 \mathrm{M}$ & 48 & $22.96 \%$ \\
\hline $2 \mathrm{M}-<4 \mathrm{M}$ & 10 & $4.78 \%$ \\
\hline $4 \mathrm{M}-6 \mathrm{M}$ & 1 & $0.47 \%$ \\
\hline Total & $\mathbf{5 9}$ & $\mathbf{2 8 . 2 2} \%$ \\
\hline
\end{tabular}

* Assessed with LVA, VA with LVA

${ }^{\wedge}$ As a percentage of the total 209 students with functional low vision 
Table 4: The following table summarizes the most common causes of visual impairment in Nepal and other developing countries:

\begin{tabular}{|c|c|c|}
\hline & Nepal - Present Study & $\begin{array}{c}\text { Other Developing Countries - } \\
\text { Recent Studies } 5,6,7,8,9\end{array}$ \\
\hline $\begin{array}{c}\text { Severe Visual Impairment / } \\
\text { Blindness }\end{array}$ & $90.85 \%$ & $\begin{array}{c}\text { Ethiopia } 92.2 \% \text {; Malawi } 83.9 \% \text {; Uganda }(76.5 \%) \\
\text { and Kenya }(66.3 \%)\end{array}$ \\
\hline $\begin{array}{l}\text { Anatomical sites of major } \\
\text { causative pathology }\end{array}$ & $\begin{array}{c}\text { Corneal scarring/phthisis } \\
(35.79 \%) \text {; Retinal disorder } \\
(20 \%) \text {,abnormalities of whole } \\
\text { globe }(13 \%) \text {, disorder of lens } \\
(12.63 \%) \text { and lesions of the } \\
\text { uvea }(1.4 \%)\end{array}$ & $\begin{array}{c}2003 \text { Study of Blind Schools in Ethiopia: Corneal } \\
\text { scarring / phthisis }(62.4 \%) \text {; cataract / aphakia } \\
(9.2 \%) \text { and lesions of the uvea }(8.8 \%) \\
2000 \text { Andhra Pradesh study in India: Retinal } \\
\text { disorders }(31.1 \%) \text {, corneal diseases }(24.3 \%), \\
\text { abnormalities of the whole globe }(20.2 \%), \\
\text { disorders of the lens }(7.9 \%) \text {, Uvea }(3.4 \%)\end{array}$ \\
\hline Corneal Visual Loss & $\begin{array}{l}\text { Corneal scarring/phthisis } \\
(35.79 \%)\end{array}$ & $\begin{array}{c}\text { Malawi (48.9\%), West Africa }(35.9 \%) \& \text { India } \\
(26.4 \%)\end{array}$ \\
\hline Etiological Categories & Childhood factors (42.11\%) & $\begin{array}{c}\text { Kenya (45.5\%), Uganda (43.4\%) and India } \\
(46.0 \%) \text {, Andhra study }(24 \%)\end{array}$ \\
\hline
\end{tabular}

Table 5: Comparison of top 5 causes of childhood blindness in 1981 and 2006

\begin{tabular}{|c|c|}
\hline 1981 Nepal Blindness Study ${ }^{10}$ & $\begin{array}{l}2006 \text { Nepal Blind Schools Study } \\
\text { (Present Study) }\end{array}$ \\
\hline Ocular Infections $21.3 \%$ & Nutritional factor (Vitamin A deficiency and measles) $15.44 \%$ \\
\hline Nutritional Causes $17.9 \%$ & Cataract / meningitis $10.18 \%$ each aggregating to $20.35 \%$ \\
\hline Congenital Cataract $16.3 \%$ & $\begin{array}{l}\text { Auto recessive hereditary factors } 8.07 \% \text { Harmful traditional eye } \\
\text { medicine } 7.37 \%\end{array}$ \\
\hline Trauma $9.0 \%$ & Glaucoma $4.53 \%$ \\
\hline Amblyopia $8.5 \%$ & Rubella $3.86 \%$ \\
\hline $80 \%$ Avoidable & 64\% Avoidable \\
\hline
\end{tabular}

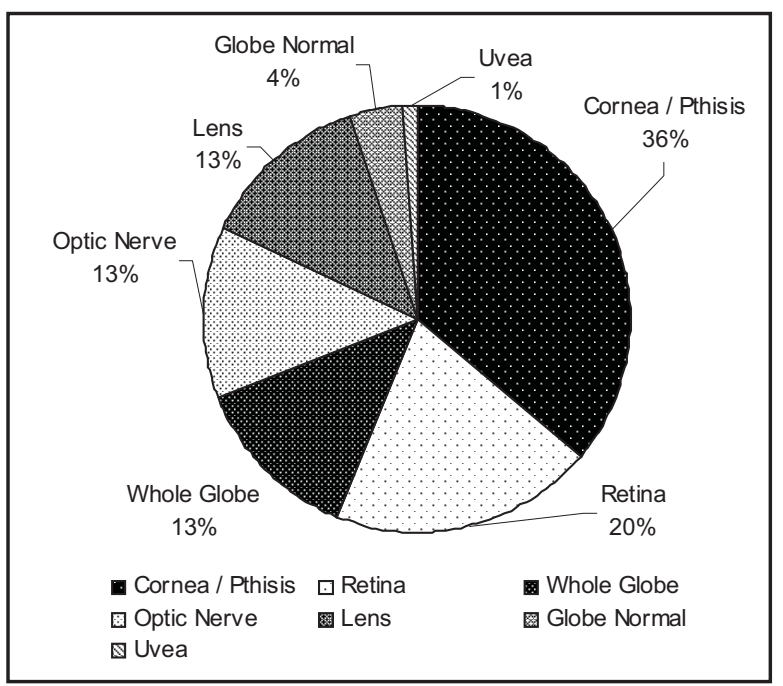

Fig 1: Major Anatomical Site of Abnormality Causing Vision Loss

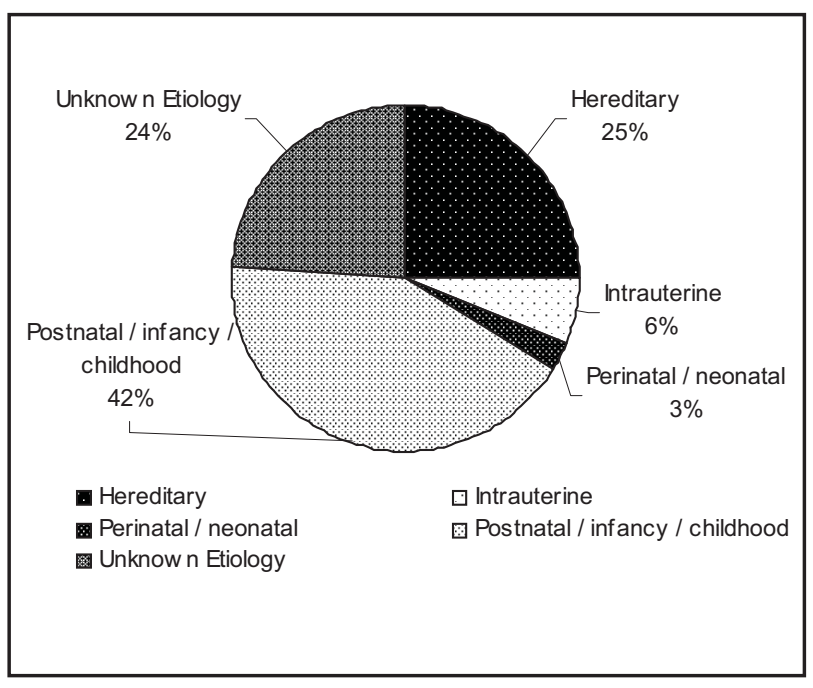

Fig 2: Etiological Categories of Low Vision and Blindness 


\section{Discussion}

A systematic search of PubMed and database shows that this is the first of its kind of study in Nepal. With an estimated blindness prevalence rate of 0.08 in 9.70 million children ${ }^{2,3}$, there are only 16 blind schools in the whole of Nepal. Total number of students enumerated in the 12 participating schools was only 345 . It is, therefore, necessary to increase number of blind schools and enrollment of visually impaired students. A national database and monitoring system for visually impaired persons must be developed and corrective action to be taken at its earliest.

Comparison with other developing countries: In many developing countries, corneal ulceration leading to corneal scar, staphyloma or phthisis is commonest cause of visual impairment leading to blindness 5 . (Table 4)

Change over Time: Since the time when the 1981 Nepal Blindness Study ${ }^{10}$ was conducted, infrastructure and human resource capacity has increased manifold and is reflected in the decrease in the proportion of avoidable childhood blindness from $80 \%$ in 1981 to $64 \%$ in 2006 . However, there could be some ambiguity in this data interpretation as the present study includes $115(40.35 \%)$ students aged above 15 years (Table 5).

Low vision status: A higher proportion of students benefited from low vision assessment in Nepal compared to a similar study of 291 students below 16 years of age done in the Andhra Pradesh district, India. Fifty seven (27.27\%) students in Nepal benefited from low vision assessment and were able to read smaller than 2M print size compared to $41(15.40 \%)$ students in Andhra Pradesh study ${ }^{9}$.

In the present study, on telescopic trial for distance visual acuity $19(9.09 \%)$ students had visual acuity better than or equal to $6 / 18$. This shows that if low vision devices for near are provided to blind children they would be able to read normal text books instead of Braille and low vision devices for distance could help them see letters on the blackboard.

In this study, twenty six $(9.11 \%)$ students who had visual acuity of $<6 / 18$ to $6 / 60$ were enrolled as blind students despite admission criteria requiring a certificate of blindness. These children were generally from weak socio-economic background and probably enrolled as blind students as they are offered free lodging, food and, oftentimes, better education.

Further research is needed to explore causal relationships between socio-economic-demographic characteristics and childhood blindness and identify risk factors so that timely and effective intervention strategies may be devised.

\section{Conclusion}

In Nepal, while there has been significant decrease in childhood blindness due to avoidable causes, much is needed to be done for the achievement of the Vision 2020 objectives with respect to childhood blindness.

Corneal opacity has been consistently found to be the most common cause of visual impairment. Eye health education should focus on, increasing awareness of using harmful traditional eye medicine (HTEM), trauma prevention, and proper nutrition of children as they are mainstay of corneal blindness.

Genetic and general eye health counseling must be made part of peri-natal and post-natal care with emphasis on follow-up. With three out of four blind children visually impaired at birth or within one year of birth, neonatal ocular screening should be made part of standard postnatal child care.

Thorough workup by ophthalmologist of visually impaired children and proper low vision assessment should be made as standard protocol for admission in blind schools.

Strong governmental and institutional support is required for capacity building, and setting up low vision rehabilitation services across Nepal. It needs to be given renewed focus with strong community participation in the detection, counseling and provision of services, in order to ensure that each visually impaired child has the best possible quality of life envisioned by the Vision 2020 .

\section{Acknowledgements}

We thank Dr. Amar Deuja, Dr. Bal Kumar K.C., Dr. Anil Serchan, Dr. HC Jha, Dr. Manoj K Sharma, Dr. Ken Bassett, Mr. Partho Banerjee, Mr. Y. D. Sapkota, Mr. Ajay Rajbhandari, Mr. Amit Dahal, Mr. Krishna Karki, Mrs. Sudha Risal Sharma and Dr. Sundip Gurung and Dr. Chet Raj Pant for their time and gracious help with this manuscript as well as to help us carry out this study.

Funding: LEI-ORBIS Pediatric Eye Care Project

\section{References}

1. Vision2020.ChildhoodBlindness[Online]. [cited 2008 July 1]. Available from: http://www.v2020. org/page.asp? section $=0001000100020005$

2. Resnikoff S, Pascolini D, Etya'ale D, Kocur I, Pararajasegaram R, Pokhrael GP, et al. Bull World Health Organ. 2004 Nov; 82(11):844-51. 
[Updated 2006 Nov 03] Available from: http:// www.who.int/bulletin/volumes/82/11/en/844. pdf

3. Central Bureau of Statistics. National Planning Commission Secretariat. Government of Nepal. Population Projection for Nepal 2002-2021. [Online]. [cited 2008 July 1]. Available from: http://www.cbs.gov.np/Population/Projection/ Table $\% 204 . h t m$

4. World Health Organization . WHO/PBL examination record for children with blindness and low vision coding instructions and manual for data entry in EPI-INFO.[Online]. [Updated 2006 July 10 ;cited 2008 July 1]; Available from: http://www.who.int/ncd/vision2020 actionplan/documents/CodingInstructions2.pdf

5. O' Sullivan J, Gilbert C, Foster A. The causes of childhood blindness in South Africa. S Afr Med J. 1997;87:1691-5.
6. Gilbert CE, Wood M, Waddel K, Foster A. Causes of childhood blindness in east Africa: results in 491 pupils attending 17 schools for the blind in Malawi, Kenya and Uganda. Ophthalmic Epidemiol. 1995;2(2):77-84.

7. Kello AB, Gilbert C. Causes of severe visual impairment and blindness in children in schools for the blind in Ethiopia. $\mathrm{Br} J$ Ophthalmol. 2003;87:526-30.

8. Sil AK, Gilbert C. Childhood Blindness in India. J Indian Med Assoc. 2001;99:100.

9. Hornby SJ, Adolph S, Gothwal VK, Gilbert CE, Dandona L, Foster A. Evaluation of children in six blind schools of Andra Pradesh. Indian $J$ Ophthalmol. 2000; 48(3):195-2.

10. Brilliant GE, editor. The epidemiology of blindness in Nepal: a report of the 1981 Nepal blindness survey. Chelsea: The Seva Foundation, 1988 .p. $141-73$ 